\title{
Design for a Continuous-Wave Focused-light Phase Plate for TEM
}

\author{
H. Müller,* Jian Jin, $†$ R. Danev, $\$$ J. Spence, $\S$ H. Padmore, $₫$ and R.M Glaeser** \\ *Physics Department, University of California, Berkeley, CA 94720, \\ $\uparrow$ Engineering Division, LBNL, University of California, Berkeley, CA 94720, \\ \$Division of Nano-Structure Physiology, Okazaki Institute for Integrative Bioscience, 5-1 Higashiyama, \\ Myodaiji, Okazaki 444-8787, Japan, \\ §Physics Department, Arizona State University, Tempe, AZ 85287-1504, \\ TAdvanced Light Source, LBNL, University of California, Berkeley, CA 94720, \\ **Life Sciences Division, LBNL, University of California, Berkeley, CA 94720.
}

It has long been known that the phase of an electron wave is shifted when the electron passes through a beam of light [1]. It has recently been proposed to employ the intense focus of a pulsed laser beam as an "electromagnetic wave" phase plate for a pulsed transmission electron microscope [2]. We now propose to use a continuous-wave (cw) laser and a resonant optical cavity as an alternative way to generate the photon density required for a quarter-wave phase plate. The cavity serves as both a focusing element and a power buildup device (Figure 1). The phase shift $\varphi$ generated during a time $\delta \mathrm{t}$ is given by $\hbar \alpha \rho \lambda \delta \eta \sqrt{m^{2}+p^{2} / c^{2}}$, where $\hbar$ is the reduced Planck constant, $\alpha$ the fine structure constant, $\rho$ the photon density, $\lambda$ the laser wavelength, $m$ the electron mass, $p$ the electron's 4-momentum, and $c$ the velocity of light. This relativistic result has been derived using the formalism of quantum electrodynamics [3]. It reduces to a similar expression found by Dawson and Fried [1] for non-relativistic electrons. The photon density of the electromagnetic field within a spherical cavity is analytically known and shown by the figure of rotation, about the vertical axis, in Figure 2A. The radial intensity distribution is shown in Figure 2B. Fabry-Perot cavities that use multilayer dielectric mirrors have been demonstrated to achieve a power buildup as large as 105 [4], but such experimental systems have so far had rather small numerical aperture (NA). In order to sufficiently limit the size of the focused light beam at the center of the cavity, a way must thus be found to increase the NA for this type of mirror. Alternatively, it is expected that large NA spherical gold mirrors can be more easily fabricated, but the reflectivity of gold is likely to limit the power buildup factor of such a cavity to no more than 50. Taking these limitations into consideration, we believe that two designs have potential to deliver sufficient power into the $2 \mu \mathrm{m}$ diameter light spot specified in Figure 1: The first would use a fully spherical gold cavity and a $5 \mu \mathrm{m}$ laser with an output power of tens of $\mathrm{W}$. The second would use a cavity consisting of a high-NA parabolic mirror and a plane mirror, which can deliver a focus as small as the one of a spherical cavity, while being easier to manufacture and accepting a collimated laser beam.

\section{References}

[1] J.W. Dawson and Z. Fried, Phys. Rev. Lett. 19 (1967) 467.

[2] See https://ipo.llnl.gov/?q=technologies-phase plate.

[3] H. Müller et al., (2010) In Preparation.

[4] H. Müller et al., Phys. Rev. Lett. 91, 020401 (2003). 


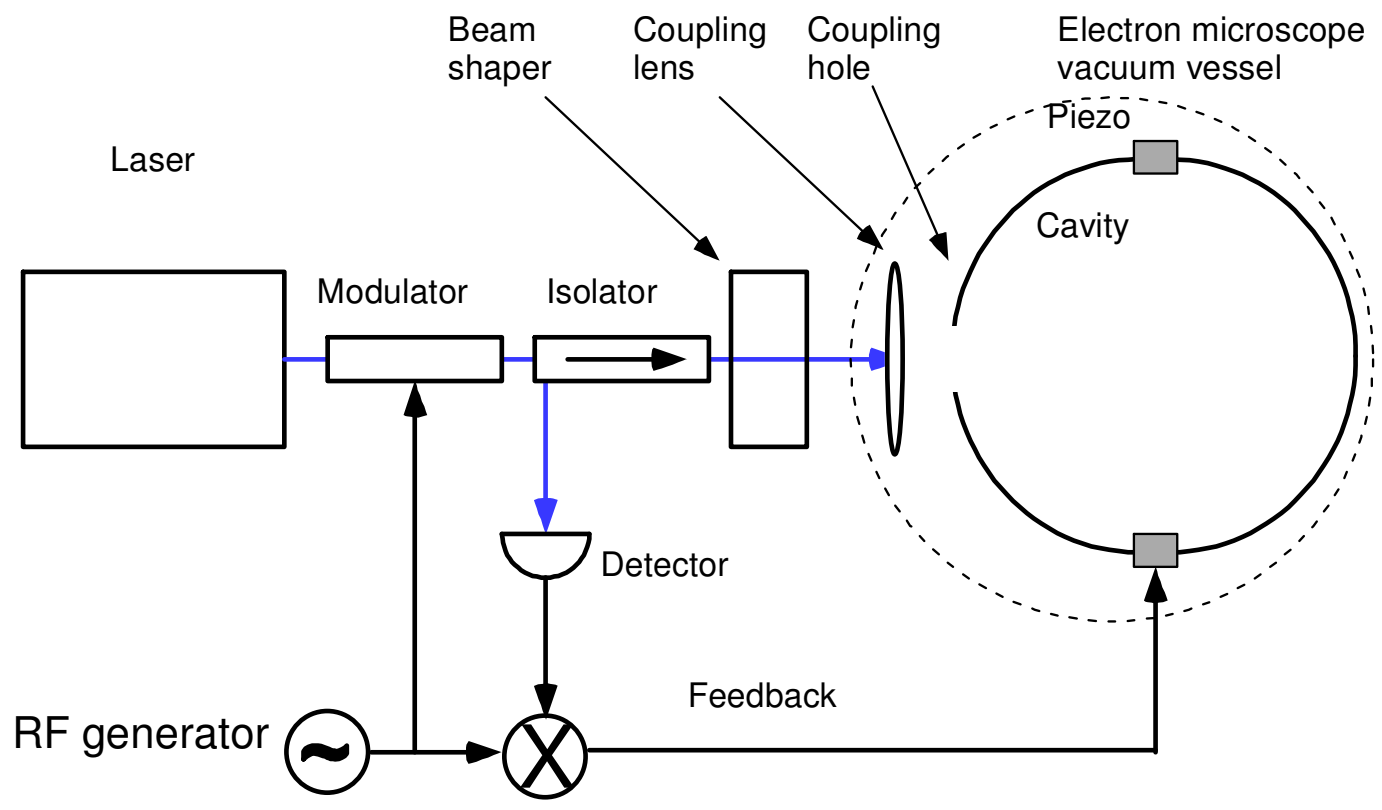

Balanced mixer

FIG. 1. Setup of the laser and optical cavity. The electron beam propagates orthogonal to the page. Its undiffracted component passes through the focus of the laser in the middle of the cavity. A focused optical beam with a diameter of $2 \mu \mathrm{m}$ in the x,y plane would provide a cut-on frequency of $1 /(40 \mathrm{~nm})$ for the phase plate, if used together with the transfer-lens system of a modified Titan 80-300 (operated at $300 \mathrm{keV}$ ), for which the effective focal length is $20 \mathrm{~mm}$.
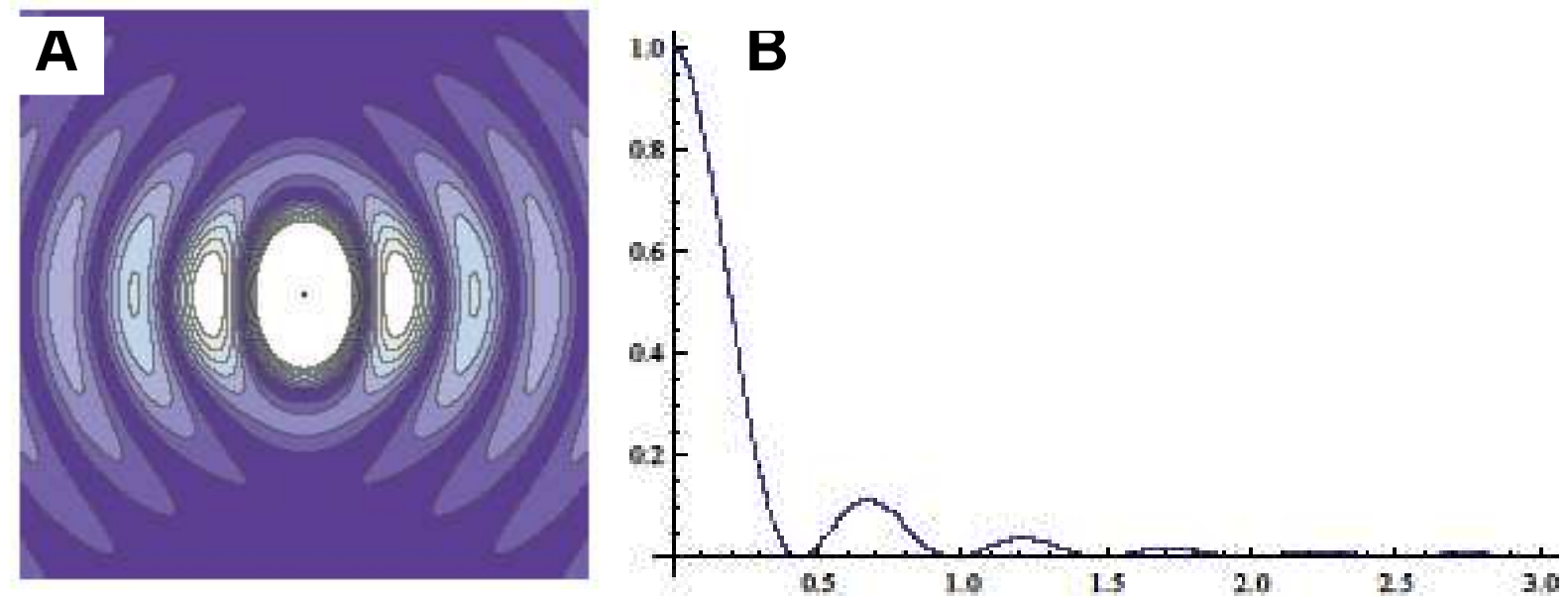

FIG. 2. (A) Contour diagram of the light intensity in the $x, z$ plane of a spherical cavity (TMn01 mode). (B) Radial light intensity in the $x, y$ plane. If the phase shift would be 90 degrees at the origin, it will be 10 degrees or less at the first subsidiary maximum 